Bundesgesundheitsbl 2020 - 63:1322-1330 https://doi.org/10.1007/s00103-020-03221-9 Eingegangen: 18. Mai 2020

Angenommen: 4. September 2020

Online publiziert: 1. Oktober 2020

(c) Der/die Autor(en) 2020

\section{Einleitung}

Seit vielen Jahren ist bekannt, dass das humane Immunsystem prinzipiell in der Lage ist, Tumorzellen zu bekämpfen. Das Immunsystem lässt sich in ein evolutionsbiologisch älteres, unspezifisches und in ein jüngeres, spezifisches oder adaptives Immunsystem aufteilen, wobei auch Interaktionen zwischen beiden Komponenten bestehen. Immuntherapeutische Ansätze sind sowohl für das unspezifische als auch für das adaptive Immunsystem seit Langem in klinischer Erforschung. Mit der Entwicklung der sogenannten Checkpointinhibitoren, die die spezifische T-Zellaktivität (adaptives Immunsystem) beeinflussen, gelang erst der eigentliche Durchbruch in der Immuntherapie. Die Immunforscher Tasuku Honjo und James Allison entdeckten mit den ersten 2 sog. Checkpoints, CTLA-4 und PD-1, ein zentrales Regulationsprinzip des Immunsystems und erhielten dafür 2018 den Nobelpreis für Medizin und Physiologie. Im Folgenden wird daher auf die T-zellregulierenden Checkpointinhibitoren eingegangen.

\section{Wirkmechanismus des adaptiven Immunsystems}

Eiweißstrukturen, die an der Tumorzelloberfläche vorkommen oder von Tumorzellen freigesetzt werden, können von Zellen des Immunsystems als fremd erkannt werden. In dem Fall werden sie Tumorantigene genannt. Um eine solche Struktur als fremd $\mathrm{zu}$ erkennen, muss sie dem Immunsystem von sogenannten

Die Autorinnen H. Zander und S. Müller-Egert teilen sich die Erstautorenschaft.

Hilke Zander · Susanne Müller-Egert · Michal Zwiewka · Steffen Groß · Ger van Zandbergen · Jörg Engelbergs

Paul-Ehrlich-Institut, Langen, Deutschland

\title{
Checkpointinhibitoren in der Tumortherapie
}

antigenpräsentierenden Zellen (APC) „Vorgestellt" werden. APCs, insbesondere dendritische Zellen (DC), können diese Tumorantigene aufnehmen und an ihrer Zelloberfläche präsentieren. Durch die Interaktion von dendritischen Zellen mit T-Helferlymphozyten über den T-Zellrezeptor (TCR) und das MHC-Klasse-II-Molekül (Major Histocompatibility Complex) werden diese antigenspezifisch stimuliert. Daraufhin vermehren sich T-Lymphozyten und wandern in die Tumorregion ein (tumorinfiltrierende Lymphozyten, TIL).

Im Tumorgewebe erkennen die T-Zellen die auf der Zelloberfläche des Tumors präsentierten Antigene über die Bindung des T-Zellrezeptors und des MHC-Klasse-I-Moleküls. Durch diese spezifische Bindung wird die zytotoxische T-Zelle (CTL) aktiviert. Sie kann die Tumorzelle hauptsächlich über die Freisetzung des porenbildenden Proteins Perforin und die Einschleusung von Granzymen in die perforierte Tumorzelle zerstören (- Abb. 1 und 2).

\section{Regulation einer Immunantwort über Checkpoints}

Immunantworten sind durch ein bemerkenswertes gegenseitiges Kontrollsystem über spezifische Kontrollpunkte, sogenannte Checkpoints reguliert [2]. Das natürliche Ziel ist das Eindämmen einer überschießenden Immunantwort, z.B. nach Eliminierung eines Fremdantigens (wie nach dem Abklingen einer viralen Infektion) oder die Vermeidung eines Angriffs auf eigene Körperzellen.

Tumoren nutzen nun diese Checkpoints auf geschickte Weise aus, um eine
Immunantwort gegen sich selbst zu verhindern.

Checkpoints regulieren sowohl die Stimulation der T-Helferzellen durch die antigenpräsentierenden Zellen als auch die Aktivierung der zytotoxischen T-Zellen (CTL). Die bisher am umfangreichsten untersuchten Checkpoints sind CTLA-4/CD80 oder CD86 und PD-1/PD-L1 oder PD-L2 (CTLA4: Cytotoxic T-Lymphocyte-associated Protein-4; PD-1: Programmed Death Protein-1; PD-L1: Programmed Death Ligand-1). Die Interaktion von CTLA-4 auf der T-Zelle mit CD80 (oder CD86) auf der antigenpräsentierenden Zelle hemmt die Stimulierung der T-Zelle durch die antigenpräsentierende Zelle (DC). Die Interaktion von PD-L1 auf der Tumorzelle mit PD-1 auf der tumorinfiltrierenden T-Zelle führt $\mathrm{zu}$ einer Hemmung der zytotoxischen Aktivität der T-Zelle (• Abb. 2).

\section{Wirkmechanismus Check- pointinhibitoren}

Mit derEntwicklung eines monoklonalen Antikörpers gegen CTLA-4 und der Zulassung von Ipilimumab in 2011 gelang ein Durchbruch in der Tumortherapie. Es konnte erstmals gezeigt werden, dass Eingriffe in das immunologische Kontrollsystem therapeutisch nutzbar sind und selbst bei fortgeschrittenen Tumorerkrankungen zu einem Langzeitüberleben führen können.

Der monoklonale Antikörper Ipilimumab bindet an CTLA-4 auf der T-Zelle. Dadurch wird die Bindung des CTLA-4 an CD80 oder CD86 auf der antigenpräsentierenden Zelle verhindert und somit die hemmende Wirkung dieser Interak- 


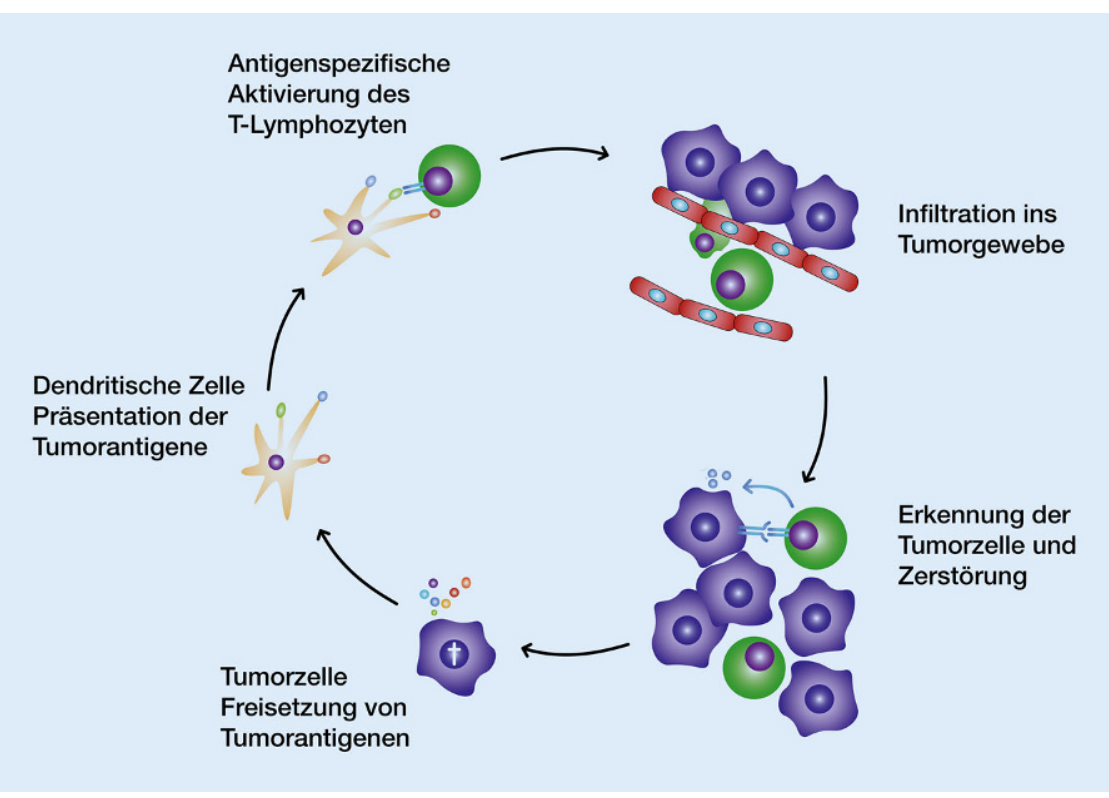

Abb. $1 \Delta$ Der zyklische Prozess der Tumorimmunität nach Chen und Mellman [1]. Am Anfang steht die Freisetzung von Tumorantigenen durch die Tumorzelle. Die Antigene werden auf der Zelloberfläche der antigenpräsentierenden Zellen (hier: dendritische Zelle) präsentiert und dadurch T-Lymphozyten aktiviert. Die aktivierten zytotoxischen T-Lymphozyten können ins Tumorgewebe einwandern und dort Tumorzellen erkennen und zerstören

tion aufgehoben. T-Zellen können dadurch wieder vermehrt durch antigenpräsentierende Zellen stimuliert werden und gegen den Tumor wirksam werden.

Durch die Entwicklung von monoklonalen Antikörpern gegen PD-1 oder PD-L1 wurde der Einsatz von immuntherapeutischen Ansätzen in der Onkologie weiter entscheidend vorangebracht. In Europa erhielten Nivolumab und kurz darauf Pembrolizumab in 2015 die Zulassung als PD-1-Inhibitoren, Atezolizumab wurde 2017 als erster PD-L1-Inhibitor zugelassen.

Die genannten Antikörper gegen PD-1 oder PD-L1 verhindern die Bindung zwischen PD-1 und PD-L1 (oder PD-L2) und stellen die gegen den Tumor gerichtete zytotoxische T-Zellantwort wieder her (•Abb. 2).

\section{Klinische Ergebnisse mit Checkpointinhibitoren}

Durch den Einsatz der Checkpointinhibitoren konnte bei Patienten mit fortgeschrittenen soliden Tumoren erstmals ein Langzeitüberleben in einem signifikanten Anteil der Patienten erzielt werden. Die $\bullet$ Abb. 3 demonstriert exemplarisch den Langzeitüberlebensvorteil ei-

\section{Probleme bei der Behandlung mit Checkpointinhibitoren}

Trotz der für die Immuntherapie charakteristischen langen Wirksamkeit spricht leider nur ein Teil der behandelten Patienten an. In klinischen Studien wurde bei soliden Tumoren eine Ansprechrate von $13 \%$ (bei Zweitlinien- oder späterer Behandlung [2L+]) bei Plattenepithelkarzinom des Kopf-Hals-Bereichs oder Urothelkarzinom, bis zu maximal $40 \%$ (bei Erstlinientherapie [1L] Melanom) oder $45 \%$ (bei 1L nichtkleinzelligem Lungenkarzinom mit einer Tumor-PDL1-Expression von $\geq 50 \%$ ) durch eine Monotherapie eines Checkpointinhibitors erreicht. Die Beurteilung eines Vorteils der Immuntherapie hängt entscheidend von der Wirksamkeit der in der jeweiligen Indikation verfügbaren Standardtherapie ab. Typischerweise ist eine Chemotherapie durch eine initial schnelle Wirksamkeit bei jedoch zumeist vergleichsweise kurzer Dauer des Ansprechens charakterisiert. Durch diese unterschiedlichen Wirkweisen kann eine Immuntherapie für einzelne Patienten gegenüber einer Standardtherapie nachteilig sein. In Studien drückt sich das durch sogenannte kreuzende Überlebenskurven aus, die einen Überlebensnachteil für die Immuntherapie gegenüber der Chemotherapie in den ersten Monaten des Beobachtungszeitraums zeigen. Dieser Kurvenverlauf lässt sich beispielweise bei dem Einsatz einer Checkpointinhibitormonotherapie bei Patienten mit vorbehandeltem, lokal fortgeschrittenem oder metastasiertem Urothelkarzinom veranschaulichen (- Abb. 4; [6]).

Leider lässt sich ein Therapieerfolg für den einzelnen Patienten bisher noch nicht ausreichend vorhersagen. Im Rahmen der klinischen Studien wird nach Biomarkern gesucht, die eine solche Vorhersage ermöglichen würden (siehe unten). Generell scheinen schlechte prognostische Faktoren wie hohe Tumorlast, rasch progredientes Tumorwachstum sowie schlechter Allgemeinzustand eher ungünstig hinsichtlich des Ansprechens einer Immuntherapie zu sein. Zudem geht man davon aus, dass Check- 
pointinhibitoren ausreichend Zeit brauchen, um wirksam werden zu können.

Durch das Einwandern von Immunzellen in die Tumorregion im Rahmen einer Immuntherapie kann es zu einer anfänglichen Vergrößerung des Tumorvolumens in den bildgebenden Verfahren kommen, die jedoch nicht Ausdruck einer echten Tumorprogression ist. Um zu vermeiden, dass dieses Phänomen einer sog. Pseudoprogression zum vorzeitigen Therapieabbruch führt, wurden spezifische Kriterien für die Bewertung des Ansprechens einer immuntherapeutischen Behandlung bei soliden Tumoren, sogenannte Immune-related-RECIST-Kriterien (iRECIST) entwickelt, die bei klinischen Hinweisen auf einen Nutzen der Therapie deren initiale Fortführung trotz Tumorvergrößerung erlauben.

Ein weiteres Problem bei der Behandlung stellen die spezifischen therapieassoziierten Nebenwirkungen dar, die sich von dem Toxizitätsprofil einer Chemotherapie unterscheiden. Durch die Verschiebung der Balance zwischen aktivierenden und hemmenden Signalen des Immunsystems können immunologisch bedingte unerwünschte Nebenwirkungen (Immune-related Adverse Events, irAEs) auftreten. Prinzipiell können alle Organe betroffen sein und klassische Autoimmunerkrankungen widerspiegeln, wie z. B. Thyreoiditis, Pneumonitis, Hepatitis oder Kolitis. Die Symptome können verzögert, selbst nach Absetzen der Therapie auftreten und im klinischen Alltag kann eine Abgrenzung gegenüber einer Progression der Grunderkrankung Schwierigkeiten bereiten $[10,11]$.

\section{PD-L1 als prädiktiver Biomarker}

In einzelnen Indikationen hat sich die Expressionsrate von PD-L1 im Tumorgewebe als Indikator für das Ansprechen einer PD-1/PD-L1-Therapie gezeigt (d.h. $\mathrm{PD}-\mathrm{L} 1$ als prädiktiver Biomarker). In den meisten Indikationen ist eine PD-L1-Expression auf Tumor- und/oder Immunzellen darüber hinaus aber auch therapieunabhängig mit dem Gesamtüberleben assoziiert (d.h. PD-L1 als prognostischer Biomarker).

Exemplarisch lässt sich das an dem Beispiel der KEYNOTE-040-Studie [12]

Bundesgesundheitsbl 2020 -63:1322-1330 https://doi.org/10.1007/s00103-020-03221-9 (c) Der/die Autor(en) 2020

\section{H. Zander · S. Müller-Egert · M. Zwiewka · S. Groß · G. van Zandbergen · J. Engelbergs Checkpointinhibitoren in der Tumortherapie}

\section{Zusammenfassung}

Mit der Entwicklung von Checkpointinhibitoren gelang in den letzten Jahren ein Durchbruch in der Tumortherapie. Checkpointinhibitoren aktivieren die Immunabwehr gegen Tumoren, indem sie die immunhemmende Wirkung spezifischer, als Kontrollpunkte agierender Zelloberflächenproteine, der sogenannten Checkpoints, aufheben. Dieser Artikel gibt einen Überblick über die Wirkweise und den Stand der derzeitigen klinischen Entwicklung zugelassener Checkpointinhibitoren. Die bisher zugelassenen Checkpointinhibitoren, gegen die Checkpoints CTLA-4 und PD-1/PD-L1 gerichtete monoklonale Antikörper, werden in verschiedenen Tumorentitäten wie Melanom, Lungen-, Nieren-, Urothelkarzinom oder Kopf-HalsTumoren sowie dem Hodgkin-Lymphom eingesetzt. Bei einem Teil dieser Patienten mit fortgeschrittenen Tumoren konnte erstmals ein Langzeitüberleben erzielt werden. In Abhängigkeit von der Tumorindikation ist diese charakteristische lange Wirksamkeit jedoch nur bei einem geringen Anteil der behandelten Patienten zu beobachten, was man durch eine Patientenselektion über prädiktive Biomarker und die Entwicklung von Kombinationstherapien zu überwinden versucht. Für manche Indikationen wurde bereits mit der Checkpointinhibitorzulassung eine Einschränkung hinsichtlich des prädiktiven PD-L1-Status vorgeschrieben.

Schlüsselwörter

Checkpointinhibitor · Immunonkologie · PD1/PD-L1 · CTLA-4 · Biomarker

\section{Checkpoint inhibitors for cancer therapy}

\begin{abstract}
In recent years, a breakthrough in tumor therapy was achieved with the development of checkpoint inhibitors. Checkpoint inhibitors activate the immune defense against tumors by overcoming the inhibitory effect of specific cell surface proteins acting as control points, the so-called checkpoints. This article provides an overview of the mode of action of approved checkpoint inhibitors and the status of current clinical development. The previously approved checkpoint inhibitors, monoclonal antibodies directed against the checkpoints CTLA-4 and PD1/PD-L1, are used in various tumor entities (including lung, kidney, and urothelial carcinoma; head and neck cancer; melanoma; and Hodgkin lymphoma). For the first time,
\end{abstract}

veranschaulichen. Patienten mit rezidivierendem oder metastasierendem Plattenepithelkarzinom der Kopf-HalsRegion(HNSCC) nach Progression unter einer vorherigen platinbasierten Therapie wurden entweder mit Pembrolizumab oder einer Standardtherapie nach Wahl des Prüfarztes (Methotrexat, Docetaxel oder Cetuximab) behandelt. Patienten mit einer Tumor-PD-L1-Expression von $\geq 50 \%$ hatten deutlich größere Überlebensvorteile von einer Behandlung mit long-term survival has been achieved in some of these patients with advanced tumors. Unfortunately, this efficacy can be observed only in a small proportion of the treated patients, depending on the tumor indication. Improved efficacy is envisioned by patient selection via predictive biomarkers and the development of combination therapies. Mandatory testing of the expression level of the predictive PD-L1 biomarker is already required in some indications to select patients with an enhanced benefit/risk relationship.

Keywords

Checkpoint inhibitor · Immuno-oncology . PD-1/PD-L1 · CTLA-4 · Biomarker

Pembrolizumab als die Patienten, in deren Tumoren sich eine niedrigere $\mathrm{PD}$ L1-Expression nachweisen ließ. Für $\mathrm{Pa}$ tienten mit PD-L1 TPS $\geq 50 \%$ zeigte sich sowohl ein besseres Ansprechen im Pembrolizumabbehandlungsarm als auch ein schlechteres Überleben im Chemotherapiearm im Vergleich zu Patienten mit PD-L1 TPS < 50\% (• Abb. 5).

Dieses unterschiedliche Ansprechen bei PD-L1 hoch und niedrig exprimierenden Tumoren hat in dem oben ge- 


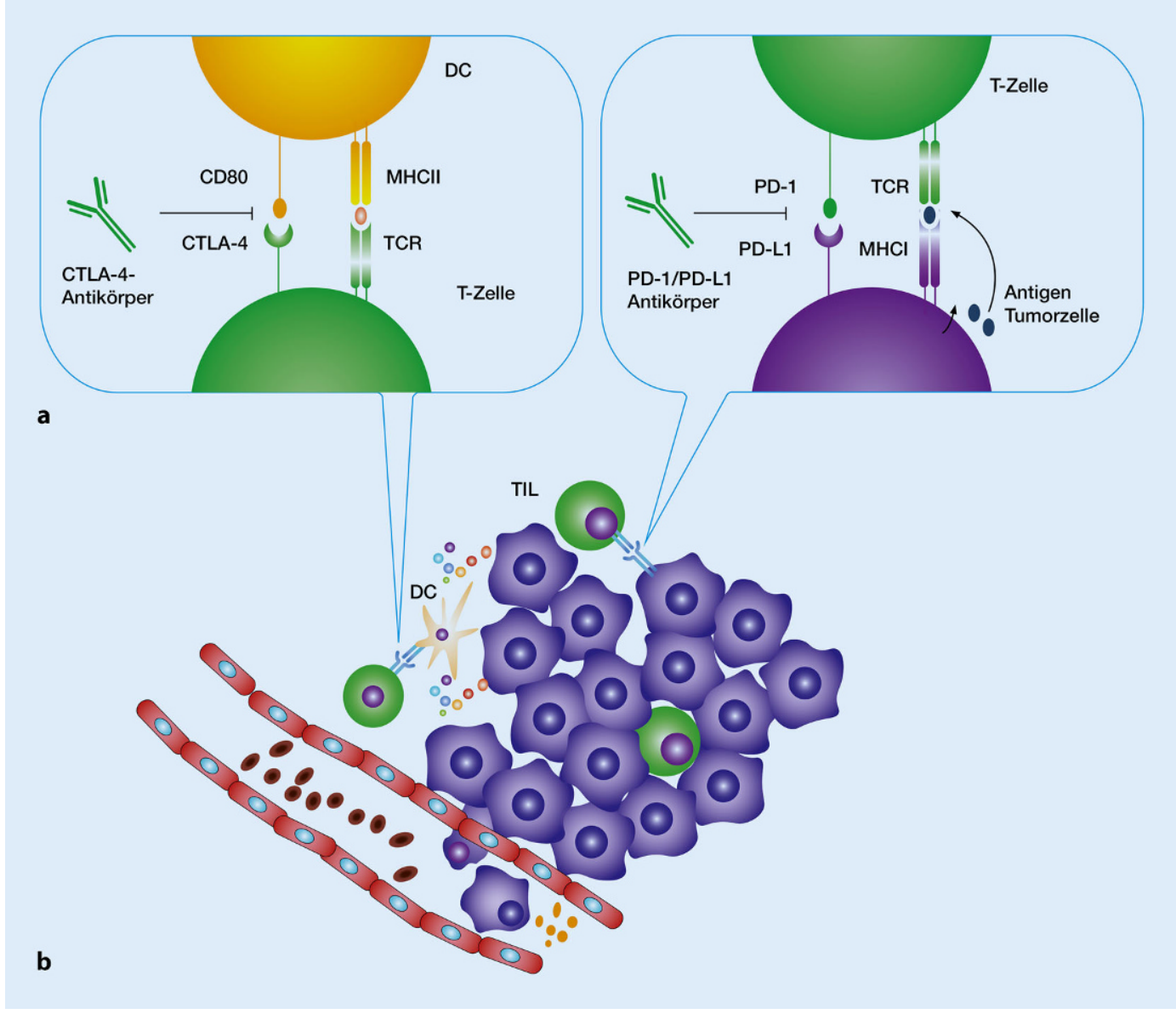

Abb. $2 \triangleleft$ Regulation der Tumorimmunität durch Checkpointinhibitoren. a T-Zellstimulierung nach Präsentation eines Tumorantigens durch eine dendritische Zelle; Hemmung der Stimulation über CTLA-4/CD80; Aufhebung der Hemmung durch CTLA-4-Antikörper; b T-Zellaktivierung nach Präsentation eines Tumorantigens durch Tumorzelle; Hemmung der T-Zellaktivität durch PD-1/PD-L1; Aufhebung der Hemmung durch PD-1/PD-L1-Antikörper

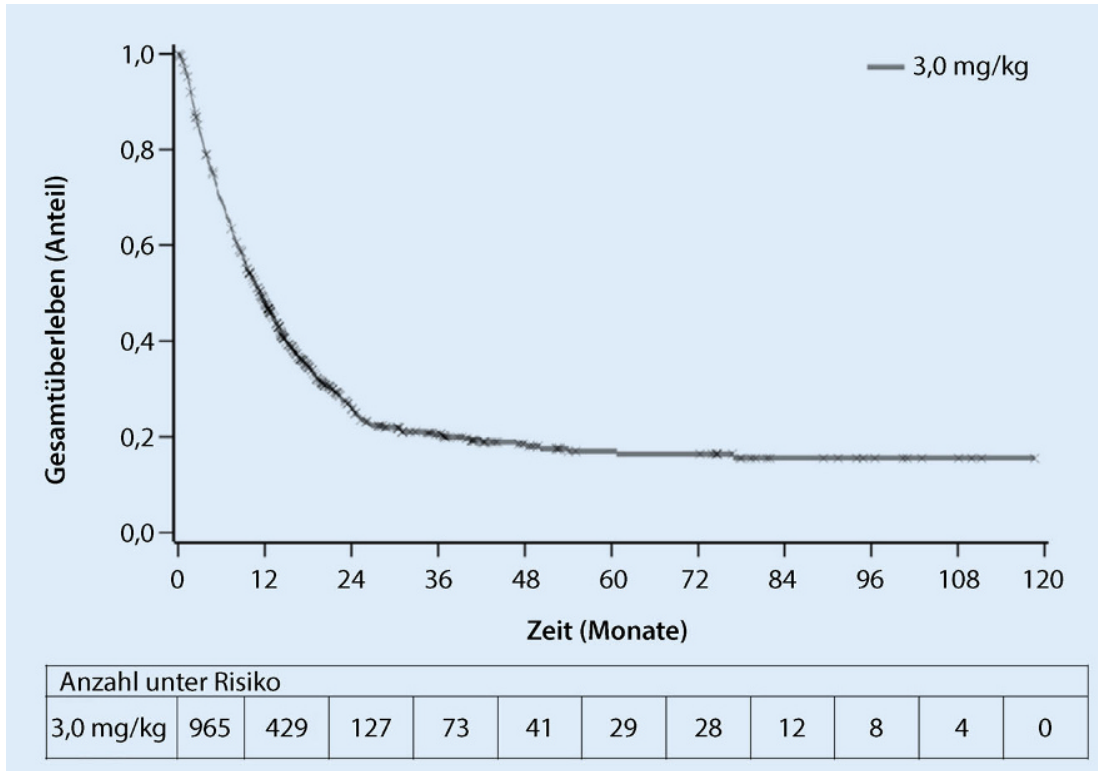

Abb. $3 \wedge$ Gesamtüberleben unter Ipilimumab (3 mg/kg) bei Patienten mit fortgeschrittenem malignen Melanom nannten Beispiel (wie auch in anderen Indikationen, • Tab. 1) zu einer Einschränkung der Zulassung für Patienten mit PD-L1 hoch exprimierenden Tumoren geführt.

In der klinischen Praxis bedeutet das, dass für Indikationen, für die eine Einschränkung hinsichtlich des PD-L1-Status besteht, die PD-L1-Expressionsstärke von Tumorproben vor dem Einsatz von Checkpointinhibitoren mit einem dafür geeigneten, analytisch validierten Test bestimmt werden muss.

\section{Behandlung mit persona-} lisierten (stratifizierten) Checkpointinhibitoren: Herausforderungen in der klinischen Praxis

In den pivotalen klinischen Studien für Checkpointinhibitoren, die nur selektiv für solche Patienten zugelassen wurden, deren Tumore eine spezifische PD-L1Expression aufwiesen, wurden checkpointinhibitor- und indikationsspezi- 
Tab. 1 Übersicht über in der EU zugelassene Checkpointinhibitoren

\begin{tabular}{|c|c|c|c|}
\hline Target & $\begin{array}{l}\text { Wirkstoff/ } \\
\text { Handelsname } \\
\text { (Zulassungsinhaber) }\end{array}$ & $\begin{array}{l}\text { Zugelassene Indikation } \\
\text { als Monotherapie }\end{array}$ & $\begin{array}{l}\text { Zugelassene Indikation } \\
\text { als Kombinationstherapie }\end{array}$ \\
\hline CTLA-4 & $\begin{array}{l}\text { Ipilimumab/Yervoy }{ }^{\circledR} \\
\text { (BMS) }\end{array}$ & Melanom & + Nivolumab in Melanom; Nierenzellkarzinom (1L) \\
\hline \multirow[t]{12}{*}{ PD-1 } & \multirow{6}{*}{$\begin{array}{l}\text { Nivolumab/Opdivo }{ }^{\circledR} \\
\text { (BMS) }\end{array}$} & Melanom & \multirow[t]{6}{*}{ + Ipilimumab in Melanom; Nierenzellkarzinom (1L) } \\
\hline & & Nichtkleinzelliges Lungenkarzinom (2L+) & \\
\hline & & Nierenzellkarzinom $(2 \mathrm{~L}+)$ & \\
\hline & & Hodgkin-Lymphom (3L+) & \\
\hline & & Plattenepithelkarzinom des Kopf-Hals-Bereichs $(2 \mathrm{~L}+)$ & \\
\hline & & Urothelkarzinom $(2 \mathrm{~L}+)$ & \\
\hline & \multirow{5}{*}{$\begin{array}{l}\text { Pembrolizumab/ } \\
\text { Keytruda }^{\circledR} \\
\text { (MSD) }\end{array}$} & Melanom & \multirow{2}{*}{$\begin{array}{l}\text { + Chemotherapie in } \\
\text { nichtkleinzelligem Lungenkarzinom (1L) }\end{array}$} \\
\hline & & $\begin{array}{l}\text { Nichtkleinzelliges Lungenkarzinom } \\
1 \mathrm{~L} \text { für PD-L1 TPS } \geq 50 \% \\
\text { 2L für PD-L1 TPS } \geq 1 \%\end{array}$ & \\
\hline & & $\begin{array}{l}\text { Plattenepithelkarzinom der Kopf-Hals-Region } \\
1 \text { L für PD-L1 CPS } \geq 1 \\
\text { 2L+: PD-L1 TPS } \geq 50 \%\end{array}$ & \multirow[t]{2}{*}{$\begin{array}{l}\text { + Chemotherapie in } \\
\text { Plattenepithelkarzinom der Kopf-Hals-Region (1L) für } \\
\text { PD-L1 CPS } \geq 1\end{array}$} \\
\hline & & Hodgkin-Lymphom (3L+) & \\
\hline & & $\begin{array}{l}\text { Urothelkarzinom } \\
2 \mathrm{~L}+\text { für alle } \\
1 \mathrm{~L} \text {, nicht für Therapie mit Cisplatin geeignet, für PD-L1 } \\
\mathrm{CPS} \geq 10\end{array}$ & + Axitinib in Nierenzellkarzinom (1L) \\
\hline & $\begin{array}{l}\text { Cemiplimab/Libtayo }{ }^{\circledR} \\
\text { (Sanofi) }\end{array}$ & Kutanes Plattenepithelkarzinom & - \\
\hline \multirow[t]{4}{*}{ PD-L1 } & \multirow{2}{*}{$\begin{array}{l}\text { Atezolizumab/ } \\
\text { Tecentriq }^{\circledR} \\
\text { (Roche) }\end{array}$} & Nichtkleinzelliges Lungenkarzinom (2L+) & \multirow[b]{2}{*}{$\begin{array}{l}\text { + Chemotherapie ( } \pm \text { Bevacizumab) in } \\
\text { nichtkleinzelligem Lungenkarzinom mit } \\
\text { nichtplattenepithelialer Histologie (1L) } \\
\text { + Chemotherapie in kleinzelligem Lungenkarzinom } \\
\text { + Chemotherapie in dreifach negativem } \\
\text { Mammakarzinom (1L) } \\
\text { für PD-L1 } 1 \% \text { (auf IC) }\end{array}$} \\
\hline & & $\begin{array}{l}\text { Urothelkarzinom } \\
2 L+\text { für alle } \\
1 \mathrm{~L} \text {, nicht für Therapie mit Cispla- } \\
\text { tin geeignet, für PD-L1 } \geq 5 \% \text { (auf IC) }\end{array}$ & \\
\hline & $\begin{array}{l}\text { Avelumab/Bavencio } \\
\text { (Merck) }\end{array}$ & Merkelzellkarzinom & + Axitinib in Nierenzellkarzinom (1L) \\
\hline & $\begin{array}{l}\text { Durvalumab/Imfinzi }{ }^{\circledR} \\
\text { (AstraZeneca) }\end{array}$ & $\begin{array}{l}\text { Nichtkleinzelliges Lungenkarzinom bei stabiler Er- } \\
\text { krankung nach platinbasierter Radiochemotherapie } \\
\text { PD-L1 } 1 \% \text { (auf TC) }\end{array}$ & - \\
\hline
\end{tabular}

fisch signifikant unterschiedliche prädiktive Schwellenwerte (Cut-offs) und Auswertungsalgorithmen (Scoring) festgelegt, um eine niedrige oder hohe PDL1-Expression zu definieren (• Tab. 1). Zudem wurden auch unterschiedliche, analytisch spezifisch validierte immunhistochemische (IHC-)Tests verwendet. Bei Pembrolizumab wurde beispielsweise der PD-L1 IHC-22C3-pharmDxTest eingesetzt (SmPC Pembrolizumab [4]), der PD-L1 sowohl auf Tumorzellen (TC) als auch auf tumorinfiltrierenden Immunzellen (IC) messen kann. Bei der Kombination von Nivolumab und
Ipilimumab wurde hingegen der PD-L1IHC-28-8-PharmDx verwendet (SmPC Nivolumab [5]), der PD-L1 nur auf TC erfassen kann. Ein Wechsel des PDL1-IHC-Testsystems während der klinischen Checkpointinhibitorentwicklung (z.B. vom Prototyp zum pivotalen Test) erfordert aufgrund der spezifischen Cutoffs und Scoringalgorithmen (TC, IC, TC+IC) umfangreiche analytische Konkordanzstudien. PD-L1-Tests wurden auch zusammen mit dem jeweiligen Checkpointinhibitor als kommerzielle CE-zertifizierte In-vitro-Diagnostika
(IVD) entwickelt und stehen für die klinische Routinetestung zur Verfügung.

Fazit: Das jeweilige Checkpointinhibitorarzneimittel und der dazugehörige spezifische prädiktive PD-L1-biomarkerbasierte IHC-Test bilden eine Art untrennbares „Tandem“. Dieses stellt eine besondere Herausforderung an die klinische Routinetestung dar, da die verfügbaren, spezifisch validierten $\mathrm{PD}$ L1-IHC-Tests aufgrund der jeweiligen PD-L1-Detektion auf unterschiedlichen Zellen (TC, IC, TC+IC) und den entsprechenden checkpointinhibitorspezifi- 


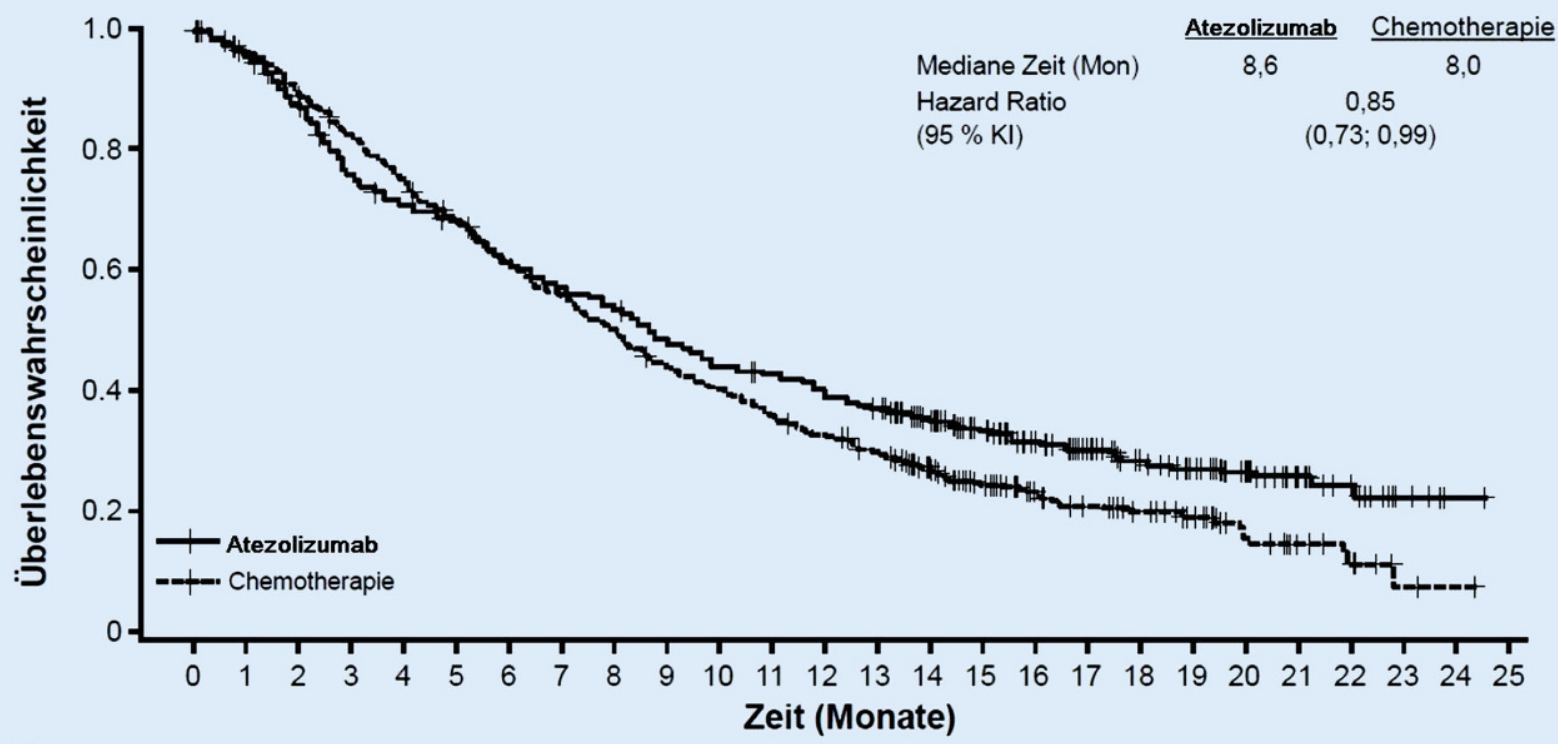

Anzahl Patienten unter

Risiko

$\begin{array}{lllllllllllllllllllllllllll}\text { Atezolizumab } & 467 & 443 & 405 & 348 & 327 & 309 & 280 & 259 & 245 & 218 & 201 & 192 & 177 & 166 & 138 & 113 & 90 & 76 & 59 & 47 & 34 & 20 & 13 & 5 & 1\end{array}$ $\begin{array}{lllllllllllllllllllllllllllllll}\text { Chemotherapie } & 464 & 428 & 397 & 364 & 330 & 299 & 268 & 244 & 219 & 191 & 175 & 156 & 140 & 126 & 99 & 78 & 60 & 49 & 42 & 30 & 17 & 11 & 7 & 2 & 1\end{array}$

Abb. 4 A Kaplan-Meier-Kurve für das Gesamtüberleben bei Patienten mit Urothelkarzinom nach Versagen einer platinhaltigen Kombinationstherapie (Studie IMvigor211). Atezolizumab: Darstellung der Überlebenskurve für die mit Atezolizumab behandelten Studienpatienten. Chemotherapie: Darstellung der Überlebenskurve für die mit Chemotherapie behandelten Studienpatienten

schen Cut-offs und Scoringalgorithmen nicht einfach austauschbar sind.

\section{Ausblick: neue prädiktive Biomarker}

Obgleich bisher die PD-L1-Expressionsstärke auf Proteinebene als prädiktiver Biomarker bei zugelassenen Checkpointinhibitoren evaluiert wurde, ist PD-L1 kein optimaler Biomarker, da die PD-L1-Expression dynamisch, heterogen und vom Zelltyp bzw. der Indikation abhängig ist und weiterhin auf unterschiedlichen IHC-Testsystemen und Cut-offs/Scoringalgorithmen beruht.

In jüngster Zeit wurden daher neue, PD-L1-unabhängige Biomarker in zahlreichen klinischen Studien hinsichtlich eines verbesserten prädiktiven Potenzials evaluiert, wie beispielsweise die Tumormutationslast (Tumor Mutational Burden, TMB) analysiert mittels Next Generation Sequencing. Hochmutierte Tumoren (d.h. mit hohem TMB) generieren mit höherer Wahrscheinlichkeit Neoantigene und können dadurch eine erhöhte Immunogenität, verbunden mit einer gesteigerten T-Zellreaktivität und antitumoralen Antwort, aufweisen. Mehrere Studien konnten bei einigen spezifischen Tumoren entsprechende prädiktive Korrelationen von hohem TMB-Wert und dem verbesserten Ansprechen auf eine Checkpointinhibitortherapie zeigen, beispielweise bei nichtkleinzelligem Bronchialkarzinom (NSCLC) für Pembrolizumab oder Atezolizumab [14, 15] beim Urothelkarzinom für Atezolizumab [16] oder bei kleinzelligem Lungenkrebs (SCLC) für Nivolumab bzw. für Nivolumab in Kombination mit Ipilimumab [17].

\section{Kombinationstherapien}

Das klinische Problem, dass bei einer Monotherapie mit Checkpointinhibitoren häufig nur wenige Patienten ansprechen, versucht man auch durch den Einsatz von Kombinationstherapien zu überwinden. Über Kombinationen von Therapien mit unterschiedlichen Wirkmechanismen möchte man eine additive, im besten Fall sogar synergistische Wirkung erreichen und/oder die Ausbildung von Resistenzen vermeiden.

Bisher zugelassen sind Kombinationen von PD-1/PD-L1-Inhibitoren mit Chemotherapie, mit CTLA-4-Inhibitor und mit Inhibitoren von vaskulären endothelialen Wachstumsfaktoren (VEGF-Inhibitoren; - Tab. 1).

\section{Kombination mit Chemotherapie}

Durch eine Chemotherapie erzielt man antiproliferative und zytotoxische Effekte. Zudem wird postuliert, dass die Tumoren z.B. durch die erhöhte Freisetzung von Antigenen empfänglicher für Immuntherapien gemacht werden könnten [18]. Zudem gibt es präklinische Hinweise darauf, dass eine Chemotherapie in manchen Fällen über die Verminderung der Anzahl der immunsuppressiv wirkenden regulatorischen T-Zellen (Tregs) die Immunantwort erhöhen könnte [19].

Tatsächlich lässt sich die Überlegenheit einer Kombination von Checkpointinhibitoren mit einer Chemotherapie in der Erstlinientherapie metastasierter Lungenkarzinome nachweisen 
TPS $\geq 50 \%$

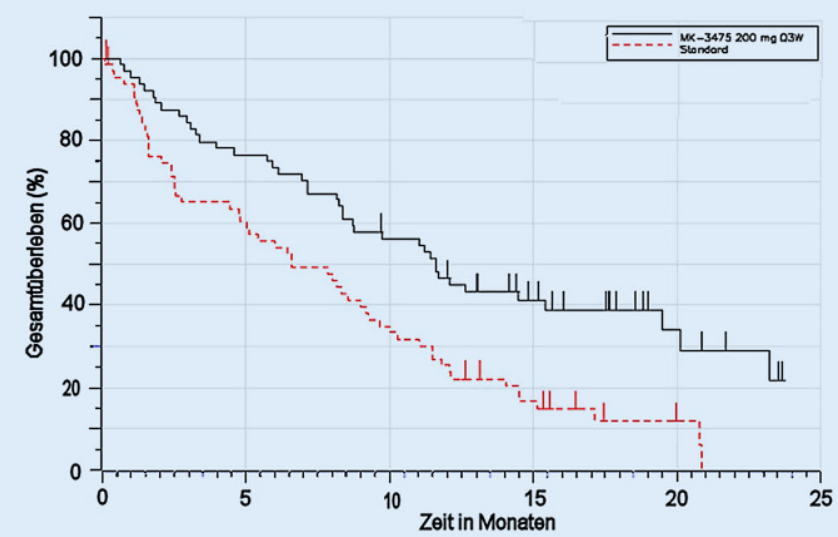

TPS $<50 \%$

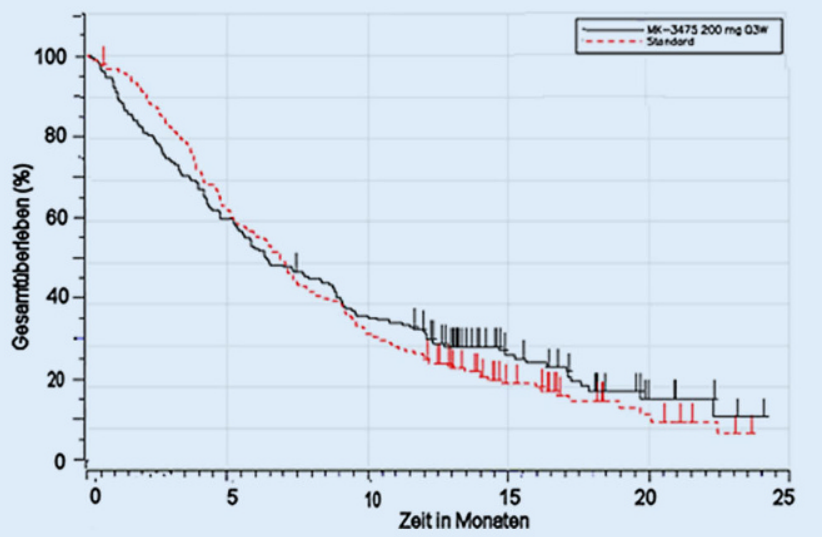

Abb. 5 ム Kaplan-Meier-Überlebenskurven für Patienten mit HNSCC in Abhängigkeit von der PD-L1-Expression im Tumorgewebe; Behandlung mit Pembrolizumab (schwarze Kurve) im Vergleich zu Chemotherapie (rote Kurve; KEYNOTE-040-Exzerpt aus [13]). Die PD-L1-Expression wurde in der KEYNOTE-040-Studie basierend auf einer qualitativen immunhistochemischen (IHC-)Methode aufProteinebene gemessen (PD-L1 IHC 22C3 pharmDxTM Test (SmPC Pembrolizumab)). TPS Tumor Proportion Score (d. h. prozentualer Anteil PD-L1-positiver Tumorzellen einer Gewebeprobe); MK-3475 Pembrolizumab

(• Tab. 1). Beispielsweise fand sich für Patienten mit nichtplattenepithelialem Lungenkarzinom eine Ansprechrate von $48 \%$ für die Kombinationstherapie im Vergleich zu 19\% für die Chemotherapie (Studie KEYNOTE-189 [20, 21]). Entsprechend zeigten sich auch für plattenepitheliale Tumore Ansprechraten von $58 \%$ vs. $38 \%$ (Studie KEYNOTE407 [22]). Demgegenüber fand sich für eine Checkpointinhibitormonotherapie eine histologieunabhängige Ansprechrate von 27,3\% (Studie KEYNOTE-042). Die Ansprechrate der Monotherapie wurde dabei vor allem durch das hohe Ansprechen der Patienten mit stark PDL1-exprimierenden Tumoren getrieben (Objective Response Rate (ORR) bei Tumor Proportion Score (TPS) $\geq 50 \%$ betrug 39,5\%; [23, 24]).

\section{Kombination mit CTLA-4}

Eine der ersten Strategien war es, eine Kombination aus Anti-CTLA-4- und Anti-PD-1-Behandlung einzusetzen, um simultan 2 komplementäre, die Immunantwort inhibierende Checkpoints auszuschalten. Kombinationen aus AntiCTLA-4- und Anti-PD-1-Therapien sind bereits im malignen Melanom und Nierenzellkarzinom zugelassen (• Tab. 1). Daten für Patienten mit fortgeschrittenem Melanom zeigten Ansprechra- ten für die Kombination Nivolumab/ Ipilimumab von 59\%, für die Nivolumabmonotherapie von $45 \%$ und $19 \%$ für die Ipilimumabmonotherapie. Die entsprechenden Gesamtüberlebensraten nach 3 Jahren betrugen $58 \%, 52 \%$ und $34 \%$ für die Kombinationstherapie bzw. für eine Monotherapie mit Nivolumab oder Ipilimumab [20]. Wichtig zu erwähnen ist hierbei, dass die Kombination von Nivolumab mit Ipilimumab nur bei Patienten mit niedriger Tumor-PD-L1Expression eine Verlängerung des Gesamtüberlebens (OS) im Vergleich zur Nivolumabmonotherapie gezeigt hat.

\section{Kombination mit VEGF-Inhibitoren}

Ebenfalls zugelassen sind Kombinationen mit Inhibitoren des vaskulären endothelialen Wachstumsfaktors (Vascular Endothelial Growth Factor, VEGF), einerseits als Tyrosinkinaseinhibitoren (z. B. Axitinib) oder als monoklonale Antikörper (z. B. Bevacizumab). Auch hier gibt es präklinische Hinweise für eine synergistische Wirkungsweise. Man vermutet, dass die VEGF-Inhibitoren eine immunsuppressive Tumorumgebung so beeinflussen können, dass eine Immunantwort gegen den Tumor ermöglicht wird [25].

Beispielsweise hat die Kombination von Avelumab und Axitinib in einer
Phase-III-Studie bei bisher unbehandelten Patienten mit fortgeschrittenem oder metastasiertem Nierenzellkarzinom eine Überlegenheit gegenüber der bisherigen Standardtherapie mit Sunitinib gezeigt (medianes progressionsfreies Überleben 13,3 vs. 8 Monate und Gesamtansprechrate $53 \%$ vs. $27 \%$ für Avelumab plus Axitinib versus Sunitinib; [7]).

\section{Ausblick: neue Kombinations- therapien}

Neben CTLA-4 und PD-1/PD-L1 sind verschiedene weitere immunologische Checkpoints auf Immunzellen vorhanden, die die Immunantwort abschwächen oder auch verstärken können. In klinischer Entwicklung sind dabei Kombinationen aus PD-1/PD-L1-Inhibitoren mit weiteren, gegen diese anderen Checkpoints gerichteten Antikörpern, die entweder, wie die bereits zugelassenen Checkpoint-Inhibitoren, die hemmenden Checkpoints blockieren (z.B. LAG-3, TIM-3) oder die Immunabwehr durch aktivierende Checkpoints direkt stimulieren (z.B. 4-1BB, OX40, CD40, GITR; [26, 27]).

Dabei sind auch Ansätze in klinischer Entwicklung, die gegen inhibitorische Moleküle auf natürlichen Killerzellen (NK-Zellen) gerichtet sind und damit auch auf die Aktivierung des unspe- 
zifischen Immunsystems zielen (z.B. gegen TIGIT gerichtete monoklonale Antikörper; [28]).

Eine weitere Kombination aus verschiedenen Immuntherapien ist der Ansatz, Checkpointinhibitoren zusammen mit Tumorvakzinen einzusetzen [29]. Das therapeutische Prinzip ist dabei, tumorspezifische Antigene "als Impfstoff“ zu nutzen und damit eine zusätzliche, gegen den Tumor gerichtete Immunantwort hervorzurufen. Das Ziel, die Immunantwort weiter zu verstärken, verfolgt man auch über die Kombinationen von Checkpointinhibitoren mit anderen Immuntherapeutika, wie bispezifischen Antikörpern (z. B. Blinatumomab) oder CAR-T-Zellen (z.B. Tisagenlecleucel-Kymriah ${ }^{\circledR} ; \quad$ Axicabtagen-Ciloleucel-Yescarta ${ }^{\circledR}$ ).

In klinischen Studien werden zudem Kombinationen von direkt in den Tumor injizierten onkolytischen Viren mit Checkpointinhibitoren evaluiert. In klinischer Entwicklung ist auch der Einsatz von zielgerichteten Therapien mit Kinaseinhibitoren (wie z. B. BRAF oder MEK) und Checkpointinhibitoren [30]. Neben diesen medikamentösen Ansätzen gibt es vorläufige klinische Daten, die auf einen möglichen Nutzen einer kombinierten Anwendung mit einer vorausgehenden oder simultanen Radiotherapie hinweisen [31].

Darüber hinaus befinden sich zahlreiche weitere Kombinationen mit Therapeutika auf Basis verschiedenster Wirkmechanismen noch in der präklinischen Entwicklung. Dies veranschaulicht, dass der mit der Zulassung der ersten Checkpointinhibitoren begonnene Wandel in der immunologischen Tumortherapie auch zukünftig fortgesetzt werden kann.

\section{Korrespondenzadresse}

\section{Dr. Jörg Engelbergs}

Paul-Ehrlich-Institut

Paul-Ehrlich-Str. 51-56, 63225 Langen,

Deutschland

joerg.engelbergs@pei.de

Funding. Open Access funding enabled and organized by Projekt DEAL.

\section{Einhaltung ethischer Richtlinien}

Interessenkonflikt. H. Zander, S. Müller-Egert, M. Zwiewka, S. Groß, G. van Zandbergen und J. Engelbergs geben an, dass kein Interessenkonflikt besteht.

Für diesen Beitrag wurden von den Autoren keine Studien an Menschen oder Tieren durchgeführt. Für die aufgeführten Studien gelten die jeweils dort angegebenen ethischen Richtlinien.

Open Access. Dieser Artikel wird unter der Creative Commons Namensnennung 4.0 International Lizenz veröffentlicht, welche die Nutzung, Vervielfältigung, Bearbeitung, Verbreitung und Wiedergabe in jeglichem Medium und Format erlaubt, sofern Sie den/die ursprünglichen Autor(en) und die Quelle ordnungsgemäß nennen, einen Link zur Creative Commons Lizenz beifügen und angeben, ob Änderungen vorgenommen wurden.

Die in diesem Artikel enthaltenen Bilder und sonstiges Drittmaterial unterliegen ebenfalls der genannten Creative Commons Lizenz, sofern sich aus der Abbildungslegende nichts anderes ergibt. Sofern das betreffende Material nicht unter der genannten Creative Commons Lizenz steht und die betreffende Handlung nicht nach gesetzlichen Vorschriften erlaubt ist, ist für die oben aufgeführten Weiterverwendungen des Materials die Einwilligung des jeweiligen Rechteinhabers einzuholen.

Weitere Details zur Lizenz entnehmen Sie bitte der Lizenzinformation auf http://creativecommons.org/ licenses/by/4.0/deed.de.

\section{Literatur}

1. Chen DS, Mellman I (2013) Oncology meets immunology: the cancer-immunity cycle. Immunity 39(1):1-10. https://doi.org/10.1016/j.immuni. 2013.07.012

2. Sharpe AH (2017) Introduction to checkpoint inhibitors and cancer immunotherapy. Immunol Rev 276(1):5-8. https://doi.org/10.1111/imr. 12531

3. SmPC Yervoy annex I summary of product characteristics. https://www.ema.europa.eu/en/ documents/product-information/yervoy-eparproduct-information_en.pdf.Zugegriffen:21.Apr. 2020

4. SMPC Keytruda annex I summary of product characteristics. https://www.ema.europa.eu/en/ documents/product-information/keytruda-eparproduct-information_en.pdf.Zugegriffen: 17. Mai 2020

5. SMPC Opdivo annex I summary of product characteristics. https://www.ema.europa.eu/en/ documents/product-information/opdivo-eparproduct-information_en.pdf.Zugegriffen: 17. Mai 2020

6. SmPC Tecentriq annex I summary of product characteristics. https://www.ema.europa.eu/en/ documents/product-information/tecentriq-eparproduct-information_en.pdf.Zugegriffen:21.Apr. 2020

7. SMPC Bavencio annex I summary of product characteristics. https://www.ema.europa.eu/ en/documents/product-information/bavencioepar-product-information_en.pdf. Zugegriffen: 17. Mai 2020
8. SMPC Imfinzi annex I summary of product characteristics. https://www.ema.europa.eu/en/ documents/product-information/imfinzi-eparproduct-information_en.pdf.Zugegriffen: 17. Mai 2020

9. SMPC Libtayo annex I summary of product characteristics. https://www.ema.europa.eu/en/ documents/product-information/libtayo-eparproduct-information_en.pdf.Zugegriffen: 17. Mai 2020

10. Haanen JBAG, Carbonnel F, Robert $C$ et al (2017) Management of toxicities from immunotherapy: ESMO Clinical Practice Guidelines for diagnosis, treatment and follow-up. Ann Oncol 28:iv119-iv142. https://doi.org/10.1093/annonc/ $\mathrm{mdx} 225$

11. Brahmer JR, Lacchetti C, Schneider BJ et al (2018) Management of immune-related adverse events in patients treated with immune checkpoint inhibitor therapy: American Society of Clinical Oncology clinical practice guideline. J Clin Oncol 36(17):1714-1768. https://doi.org/10.1200/JCO. 2017.77.6385

12. Cohen EEW, Soulières D, Le Tourneau C et al (2019) Pembrolizumab versus methotrexate, docetaxel, or cetuximab for recurrent or metastatic headand-neck squamous cell carcinoma (KEYNOTE040): a randomised, open-label, phase 3 study. Lancet 393(10167):156-167. https://doi.org/10. 1016/S0140-6736(18)31999-8

13. Epar Keytruda (2020) Assessment report Keytruda. EMA/CHMP/591139/2019/corr. https://www. ema.europa.eu/en/documents/variation-report/ keytruda-h-c-3820-ii-0065-epar-assessmentreport-variation_en.pdf. Zugegriffen: 17 . Mai 2020

14. RizviNA, HellmannMD, Snyder Aetal (2015)Cancer immunology. Mutational landscape determines sensitivity to PD-1 blockade in non-small cell lung cancer. Science 348(6230):124-128. https://doi. org/10.1126/science.aaa1348

15. Gandara DR, Paul SM, Kowanetz M et al (2018) Blood-based tumor mutational burden as a predictor of clinical benefit in non-small-cell lung cancer patients treated with atezolizumab. Nat Med 24(9):1441-1448. https://doi.org/10.1038/ s41591-018-0134-3

16. Rosenberg JE, Hoffman-Censits J, Powles T et al (2016) Atezolizumab in patients with locally advanced and metastatic urothelial carcinoma who have progressed following treatment with platinum-based chemotherapy: a single-arm, multicentre, phase 2 trial. Lancet 387(10031):1909-1920. https://doi.org/10.1016/ S0140-6736(16)00561-4

17. Hellmann MD, Paz-Ares L, Bernabe Caro R et al (2019) Nivolumab plus Ipilimumab in advanced non-small-cell lung cancer. N Engl J Med 381(21):2020-2031. https://doi.org/10.1056/ NEJMoa1910231

18. Zitvogel L, Kepp O, Kroemer G (2011) Immune parameters affecting the efficacy of chemotherapeutic regimens. Nat Rev Clin Oncol 8(3):151-160. https://doi.org/10.1038/nrclinonc.2010.223

19. Włodarczyk M, Ograczyk E, Kowalewicz-Kulbat M, Druszczyńska M, Rudnicka W, Fol M (2018) Effect of cyclophosphamide treatment on central and effector memory $\mathrm{T}$ cells in mice. Int J Toxicol 37(5):373-382. https://doi.org/10.1177/ 1091581818780128

20. Wolchok JD, Chiarion-Sileni V, Gonzalez R et al (2017) Overall survival with combined nivolumab and ipilimumab in advanced melanoma. $\mathrm{N}$ Engl J 


\section{Leitthema}

Med 377(14):1345-1356. https://doi.org/10.1056/ NEJMoa1709684

21. Gandhi L, Rodríguez-Abreu D, Gadgeel $S$ et al (2018) Pembrolizumab plus chemotherapy in metastatic non-small-cell lung cancer. N Engl J Med 378(22):2078-2092. https://doi.org/10.1056/ NEJMoa1801005

22. Paz-AresL,LuftA, VicenteDetal (2018) Pembrolizumab plus chemotherapy for squamous non-smallcell lung cancer. N Engl J Med 379(21):2040-2051. https://doi.org/10.1056/NEJMoa1810865

23. Mok TSK, Wu Y-L, Kudaba I et al (2019) Pembrolizumab versus chemotherapy for previously untreated, PD-L1-expressing, locally advanced or metastatic non-small-cell lung cancer (KEYNOTE042): a randomised, open-label, controlled, phase 3 trial. Lancet 393(10183):1819-1830. https://doi. org/10.1016/S0140-6736(18)32409-7

24. Reck M, Rodríguez-Abreu D, Robinson AG et al (2016) Pembrolizumab versus chemotherapy for PD-L1-positive non-small-cell lung cancer. N Engl J Med 375(19):1823-1833. https://doi.org/10.1056/ NEJMoa1606774

25. Fukumura D, Kloepper J, Amoozgar Z, Duda DG, Jain RK (2018) Enhancing cancer immunotherapy using antiangiogenics: opportunities and challenges. Nat Rev Clin Oncol 15(5):325-340. https://doi. org/10.1038/nrclinonc.2018.29

26. Marin-Acevedo JA, Dholaria B, Soyano $A E$, Knutson KL, Chumsri S, Lou Y (2018) Next generation of immune checkpoint therapy in cancer: new developments and challenges. JHematol Oncol 11(1):39. https://doi.org/10.1186/ s13045-018-0582-8

27. Mahoney KM, Rennert PD, Freeman GJ (2015) Combination cancer immunotherapy and new immunomodulatory targets. Nat Rev Drug Discov 14(8):561-584. https://doi.org/10.1038/nrd4591

28. Manieri NA, Chiang EY, Grogan JL (2017) TIGIT: a key inhibitor of the cancer immunity cycle. Trends Immunol 38(1):20-28. https://doi.org/10.1016/j. it.2016.10.002

29. OttPA, HuZ,KeskinDBetal (2017)Animmunogenic personal neoantigen vaccine for patients with melanoma. Nature 547(7662):217-221. https:// doi.org/10.1038/nature22991

30. Cyprian FS, Akhtar S, Gatalica Z, Vranic S (2019) Targeted immunotherapy with a checkpoint inhibitor in combination with chemotherapy: a new clinical paradigm in the treatment of triplenegative breast cancer. Bosn J Basic Med Sci 19(3):227-233. https://doi.org/10.17305/bjbms. 2019.4204

31. Ko EC, Formenti SC (2018) Radiotherapy and checkpoint inhibitors: a winning new combination? Ther Adv Med Oncol 10:1758835918768240. https:// doi.org/10.1177/1758835918768240 